\title{
Lewis Acid Free High Speed Synthesis of Nimesulide-Based Novel $N$-Substituted Cyclic Imides
}

\author{
Kavitha Kankanala, ${ }^{a, b}$ Vangala Ranga Reddy, ${ }^{c}$ Khagga Mukkantia and Sarbani Pal ${ }^{*, b}$ \\ ${ }^{a}$ JNT University Hyderabad, Kukatpally, Hyderabad-500085, India \\ ${ }^{b}$ Department of Chemistry, MNR Degree and PG College, Kukatpally, Hyderabad-500072, India \\ 'Dr. Reddy's Laboratories Ltd. Integrated Product Development, Bachupally, \\ Hyderabad-500090, India
}

\begin{abstract}
A primeira síntese de novas imidas cíclicas derivadas de nimessulida foi realizada via reação de uma imina preparada a partir de nimessulida com anidridos apropriados na presença de acetato de sódio. Usando este processo, uma variedade de imidas cíclicas N-substituídas foi preparada em bons rendimentos em ácido acético glacial. Alguns dos compostos sintetizados mostraram atividades anti-inflamatórias quando testados in vivo.
\end{abstract}

The first synthesis of nimesulide-based novel cyclic imides has been accomplished via the reaction of an amine prepared from nimesulide with appropriate anhydrides in the presence of sodium acetate. Using this process a variety of $N$-substituted cyclic imides was prepared in good yields in glacial acetic acid. Some of the compounds synthesized showed anti-inflammatory activities when tested in vivo.

Keywords: nimesulide, anhydride, cyclic imide, anti-inflammatory activities

\section{Introduction}

$\mathrm{N}$-Substituted cyclic imides A (Figure 1) represent an important class of bioactive molecules that show a wide range of pharmacological activities such as androgen receptor antagonistic, anti-inflammatory, anxiolytic, antiviral, antibacterial, and antitumor properties. ${ }^{1-11}$ On the other hand $\mathrm{N}$-(4-nitro-2-phenoxy phenyl) methanesulfonamide or nimesulide B (see Figure 1), a preferential cyclooxygenase-2 (COX-2) inhibitor is one of the well known non-steroidal anti-inflammatory drugs (NSAIDs) that has been utilized to treat pain and other inflammatory diseases. Because of their common antiinflammatory properties and our interest in nimesulide derivatives $^{12}$ as potential anti-inflammatory agents we decided to prepare compound $\mathbf{C}$ having structural features of both $\mathbf{A}$ and $\mathbf{B}$.

In spite of their extensive pharmaceutical and industrial use only a limited number of procedures are available for the synthesis of $\mathbf{A} .^{13,14}$ These include (i) the dehydrative condensation of an anhydride and an amine at high

*e-mail: sarbani277@yahoo.com

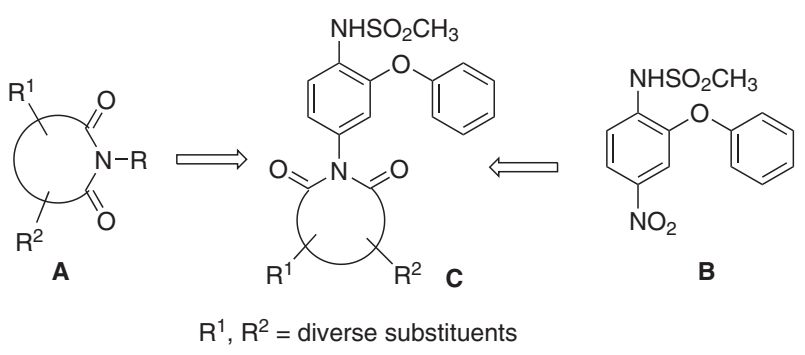

Figure 1. Design of novel cyclic imide derived from nimesulide.

temperature, ${ }^{15}$ (ii) the cyclization of the amic acid in the presence of acidic reagents, ${ }^{16}$ (iii) $\mathrm{N}$-alkylation of maleimide with alcohols under Mitsunobu reaction conditions, ${ }^{13}$ (iv) Lewis acids or hexamethyldisilazane catalyzed synthesis of $N$-alkyl and $N$-arylimide derivatives ${ }^{17}$ and dehydrative cyclization of acid anhydrides, imides and dicarboxylic acids with substituted amines in the presence of DPPOx and $\mathrm{Et}_{3} \mathrm{~N} .{ }^{18}$ However, many of these methodologies suffer from several drawbacks such as (i) the use of expensive catalysts or Lewis acids and carcinogenic solvent media and (ii) formation of numerous by-products leading to the poor yields of products. Moreover, only a narrow range of imide derivatives can be synthesized by using these methods. 
While microwave assisted addition of amines to phthalic anhydride has been studied, ${ }^{19}$ no significant difference was observed when the reaction was carried out by microwave or conventional heating in DMF. ${ }^{20}$ Moreover, microwaveassisted solvent free synthesis of cyclic imides required the use of $\mathrm{TaCl}_{5}$-silica gel as catalyst. ${ }^{21}$ To overcome all these issues, we herein report the Lewis acid free rapid synthesis ${ }^{22}$ of novel $N$-substituted cyclic imides derived from nimesulide under conventional heating.

\section{Results and Discussion}

The starting compound $\mathbf{1}$ required for our study was prepared $^{12}$ in quantitative yield from nimesulide B via reducing its nitro group as shown in Scheme 1.

The aromatic amine was then treated with a variety of cyclic anhydrides 2 (Scheme 1) and the results are summarized in Table 1. In the beginning of our study, the reaction of aromatic amine $\mathbf{1}$ with phthalic anhydride 2a was examined under a conventional condition. After refluxing the reaction mixture in glacial acetic acid for $1.5 \mathrm{~h}$ the desired imide $3 \mathbf{a}$ was isolated in $62 \%$ yield (Entry 1, Table 1). In order to improve the product yield the above reaction was carried out in the presence of $\mathrm{NaOAc}$ in the same solvent.

The reaction was completed within 10 min affording 3a in $73 \%$ yield (Entry 2, Table 1). A further accelerating effect was observed when the reaction was carried out in the presence of $\mathrm{NaOAc}$ providing 3a in 62 and $73 \%$ yield respectively (Entry 1 and 2, Table 1). Encouraged by these observations, especially in the presence of $\mathrm{NaOAc}$ (Entry 2, Table 1) when the reaction was completed within $10.0 \mathrm{~min}$, we decided to asses the generality of this process for the synthesis of other related derivatives. As indicated in Table 1 that nitro substituted phthalic anhydrides (2b and $\mathbf{2 c}$ ) reacted with $\mathbf{1}$ even slower than $\mathbf{2 a}$ (Entries 3 and 4 vs 2, Table 1) in the presence of sodium acetate. Among the aliphatic anhydrides, the six-membered anhydride 2f (Entry 7, Table 1) produced the best result. All these reactions were also performed under conventional heating condition without sodium acetate (c.f. Entry 1, Table 1) and the results were compared with that of sodium acetate
Table 1. Synthesis of novel $N$-substituted cyclic imides

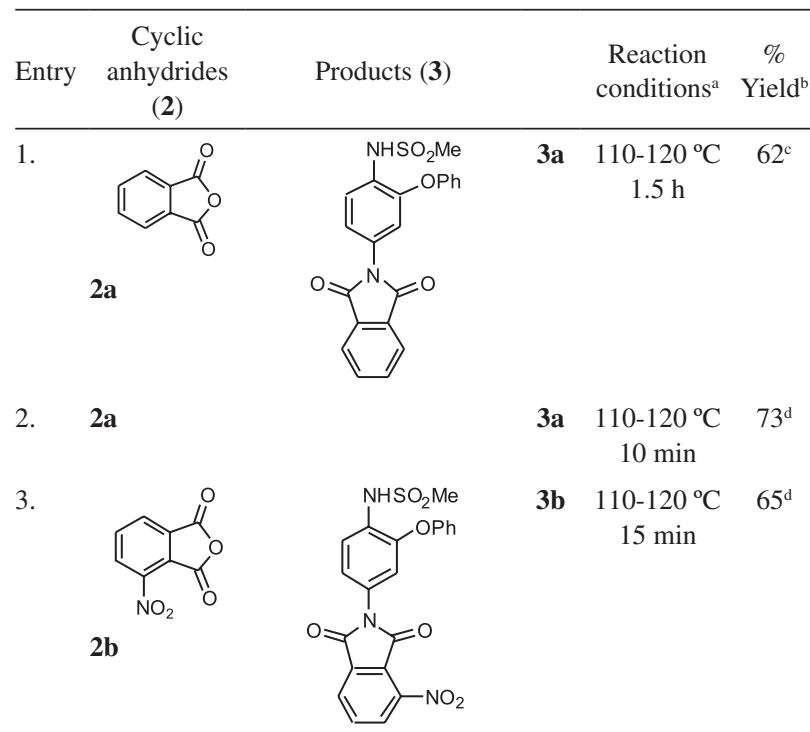

4.<smiles>O=C1COC2(COC(=O)c3cc([N+](=O)[O-])ccc32)O1</smiles>

$2 \mathrm{c}$<smiles>O=C1c2ccc([N+](=O)[O-])cc2C(=O)N1c1ccc([N+](=O)[O-])c(O)c1</smiles>

3c $\quad 110-120^{\circ} \mathrm{C} \quad 65^{\mathrm{d}}$ $15 \mathrm{~min}$

3d $\quad 110-120^{\circ} \mathrm{C} \quad 65^{\mathrm{d}}$ $15 \mathrm{~min}$

5.<smiles>O=C1C=CC(=O)N1c1ccc([N+](=O)[O-])c(O)c1</smiles>

6.<smiles>O=C1CCC(=O)O1</smiles><smiles>O=C1CCC(=O)N1c1ccc([N+](=O)[O-])c(O)c1</smiles>

3e $110-120^{\circ} \mathrm{C} \quad 65^{\mathrm{d}}$ $20 \mathrm{~min}$<smiles>O=C1CCCC(=O)O1</smiles><smiles>O=C1CCCC(=O)N1c1ccc([N+](=O)[O-])cc1</smiles>

3f $\quad 110-120^{\circ} \mathrm{C} \quad 75^{\mathrm{d}}$ $20 \mathrm{~min}$

${ }^{\mathrm{a}} \mathrm{All}$ the reactions were carried out using compound $\mathbf{1}(1.0 \mathrm{mmol})$, cyclic anhydride $2(1.0 \mathrm{mmol})$ and anhydrous NaOAc $(100 \mathrm{mg}, 1.2 \mathrm{mmol})$. ${ }^{b}$ Isolated yield. ${ }^{\mathrm{c}}$ The reaction was carried out in the absence of $\mathrm{NaOAc}$. ${ }^{\mathrm{d}}$ Glacial acetic acid was used as a solvent.<smiles>COS(=O)(=O)c1ccc([N+](=O)[O-])cc1Oc1ccccc1</smiles>

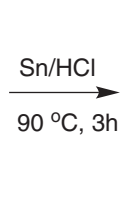<smiles>COS(=O)(=O)c1ccc(N)cc1Oc1ccccc1</smiles><smiles>CCCCC(=O)OC(=O)OCC</smiles><smiles>COS(=O)(=O)c1ccc(N2C(=O)CCCCCC2=O)cc1Oc1ccccc1</smiles>

Scheme 1. Preparation of $N$-substituted cyclic imides from nimesulide. 
catalysed method. Nevertheless, all the compounds prepared are novel and well characterized by spectral and analytical data.

Having synthesized a range of nimesulide-based novel cyclic imides we decided to examine anti-inflammatory activities of selected compounds e.g. 3a, 3b, 3c, 3e and 3f. The anti-inflammatory activity of these compounds was evaluated in a carrageenan-induced rat model of inflammation ${ }^{23}$ using indomethacin as a reference compound. At a dose of $100 \mathrm{mg} \mathrm{kg}^{-1}$ (i.p.) compound $\mathbf{3 f}$ showed 20, 25, 45 and 40\% inhibition of edema after 1,2, 3 and $4 \mathrm{~h}$ when indomethacin showed 24, 43, 66 and 93\% inhibition at $10 \mathrm{mg} \mathrm{kg}^{-1}$ at the same time points. Though the other compounds were also found to be active (15-25\% inhibition of edema at various time points) when dosed at $100 \mathrm{mg} \mathrm{kg}^{-1}$ the compound $\mathbf{3 f}$ was found to be the best among them.

\section{Conclusions}

We have described a simple and rapid synthesis of novel cyclic substituted imides ${ }^{24}$ that were designed from a well known drug nimesulide. The aromatic amine prepared from nimesulide was reacted with a variety of cyclic anhydrides in the presence of sodium acetate to afford the desired products within few minutes. Overall, due to the shorter reaction time and simple operational procedure that does not require the use of expensive catalysts / solvents, would find wide application in the synthesis of various nimesulidebased cyclic imides of potential pharmacological interest. Some of the compounds synthesized showed antiinflammatory activity when tested in rats.

\section{Experimental}

Melting points were determined by open glass capillary method on a Cintex melting point apparatus and are uncorrected. IR spectra were recorded on a Perkin-Elmer spectrometer using $\mathrm{KBr}$ pellets. ${ }^{1} \mathrm{HNMR}$ spectra were recorded on a Bruker ACF-300 machine and a Varian 300 and $400 \mathrm{MHz}$ spectrometer using $\mathrm{CDCl}_{3}$ or DMSO- $d_{6}$, with reference to tetramethylsilane as an internal reference. ${ }^{13} \mathrm{CNMR}$ spectra were recorded on a $75 \mathrm{MHz}$ spectrometer. Elemental analyses were performed by Varian 3LV analyzer series $\mathrm{CHN}$ analyzer. Mass spectra were recorded on a Jeol JMC D-300 instrument by using Electron ionization at $70 \mathrm{ev}$. All reactions were monitored by TLC on pre-coated silica gel plates. Column chromatography was performed on 100-200 mesh silica gel (SRL, India) using 10-20 times (by weight) of the crude product. All the anhydrides used are commercially available.

\section{Preparation of cyclic amides (3)}

\section{Typical procedure for the preparation of $3 a$}

To a mixture of compound $\mathbf{1}(278 \mathrm{mg}, 1.0 \mathrm{mmol})$ and phthalic anhydride $2 \mathbf{a}(148 \mathrm{mg}, 1.0 \mathrm{mmol})$ in glacial acetic acid $(3 \mathrm{~mL})$ was added anhydrous sodium acetate $(100 \mathrm{mg}$, $1.2 \mathrm{mmol}$ ) and the mixture was allowed to reflux for $10 \mathrm{~min}$. After completion of the reaction (as indicated by TLC) the mixture was added to crushed ice $(50 \mathrm{~g})$ and stirred. The solid separated was filtered and dried. The crude product was purified by column chromatography followed by recrystallization from methanol.

N-[4-(1,3-dioxo-1,3-dihydroisoindol-2-yl)-2-phenoxyphenyl]methanesulfonamide (3a)

White solid; mp $230-232{ }^{\circ} \mathrm{C} ; \mathrm{R}_{\mathrm{f}} 0.95\left(\mathrm{CHCl}_{3}\right.$ : Ethylacetate $=9: 1), \mathrm{IR}(\mathrm{KBr}) v_{\max } / \mathrm{cm}^{-1}: 1714,{ }^{1} \mathrm{HNMR}$ $\left(400 \mathrm{MHz}, \mathrm{CDCl}_{3}\right) \delta 3.0(\mathrm{~s}, 3 \mathrm{H}), 6.8(\mathrm{~m}, 2 \mathrm{H}), 7.1(\mathrm{~m}, 2 \mathrm{H})$, $7.2(\mathrm{~m}, 2 \mathrm{H}), 7.4(\mathrm{~m}, 2 \mathrm{H}), 7.7(\mathrm{~m}, 3 \mathrm{H}), 7.9(\mathrm{~m}, 2 \mathrm{H}) ;{ }^{13} \mathrm{CNMR}$ $\left(75 \mathrm{MHz}, \mathrm{CDCl}_{3}\right) \delta 40.0\left(\mathrm{CH}_{3}\right), 116.1(\mathrm{CH}), 118.9(\mathrm{CH})$, $121.1(\mathrm{CH}), 122.1(\mathrm{CH}), 123.8(\mathrm{CH}), 124.8(\mathrm{CH}), 127.6$ (C), $128.6(\mathrm{C}), 130.2(\mathrm{CH}), 131.5(\mathrm{C}), 134.5(\mathrm{CH}), 147.2$ (C), $155.2(\mathrm{C}), 166.8(\mathrm{C})$; MS $408\left(\mathrm{M}^{+}, 100 \%\right)$; Elemental analysis found: $\mathrm{C}, 61.70, \mathrm{H} 3.94, \mathrm{~N} 6.89 ; \mathrm{C}_{21} \mathrm{H}_{16} \mathrm{~N}_{2} \mathrm{O}_{5} \mathrm{~S}$ requires $\mathrm{C}, 61.75 ; \mathrm{H}, 3.95 ; \mathrm{N}, 6.86 \%$.

$\mathrm{N}$-[4-(4-nitro-1,3-dioxo-1,3-dihydroisoindol-2-yl)-2-phenoxyphenyl]methanesulfonamide ( $\mathbf{3} \boldsymbol{b})$

Light green solid; $\mathrm{mp} 214-216{ }^{\circ} \mathrm{C} ; \mathrm{R}_{\mathrm{f}} 0.68\left(\mathrm{CHCl}_{3}\right.$ : Ethylacetate $=9: 1) ; \mathrm{IR}(\mathrm{KBr}) v_{\max } / \mathrm{cm}^{-1}: 1732 ;{ }^{1} \mathrm{HNMR}$ $\left(400 \mathrm{MHz}, \mathrm{DMSO}-d_{6}\right) \delta 3.0(\mathrm{~s}, 3 \mathrm{H}), 7.0(\mathrm{~s}, 1 \mathrm{H}), 7.1(\mathrm{~d}$, $J 7.8 \mathrm{~Hz}, 2 \mathrm{H}), 7.2(\mathrm{~m}, 2 \mathrm{H}), 7.4(\mathrm{t}, J 7.8 \mathrm{~Hz}, 2 \mathrm{H}), 7.6$ (d, $J 8.3 \mathrm{~Hz}, 1 \mathrm{H}), 8.0(\mathrm{t}, J 7.8 \mathrm{~Hz}, 1 \mathrm{H}), 8.2(\mathrm{~d}, J 6.7 \mathrm{~Hz}, 1 \mathrm{H})$, 8.3 (d, J $7.4 \mathrm{~Hz}, 1 \mathrm{H}), 9.5$ (bs, $1 \mathrm{H}, \mathrm{NH}) ;{ }^{13} \mathrm{CNMR}(75 \mathrm{MHz}$, $\left.\mathrm{CDCl}_{3}\right) \delta 39.9\left(\mathrm{CH}_{3}\right), 116.0(\mathrm{CH}), 119.0(\mathrm{CH}), 120.8(\mathrm{CH})$, $122.2(\mathrm{CH}), 125.0(\mathrm{CH}), 127.4(\mathrm{CH}), 127.6(\mathrm{C}), 128.3(\mathrm{CH})$, $128.9(\mathrm{C}), 130.3(\mathrm{CH}), 135.8(\mathrm{CH}), 147.2(\mathrm{C}), 155.1(\mathrm{C}) ; \mathrm{MS}$ $453\left(\mathrm{M}^{+}, 100 \%\right)$; Elemental analysis found: $\mathrm{C}, 55.69, \mathrm{H} 3.35$, $\mathrm{N} 9.19 ; \mathrm{C}_{21} \mathrm{H}_{15} \mathrm{~N}_{3} \mathrm{O}_{7} \mathrm{~S}$ requires $\mathrm{C}, 55.63 ; \mathrm{H}, 3.33 ; \mathrm{N}, 9.27 \%$.

N-[4-(5-nitro-1,3-dioxo-1,3-dihydroisoindol-2-yl)-2phenoxyphenyl]methanesulfonamide (3c)

Light yellow solid; mp 192-194 ${ }^{\circ} \mathrm{C} ; \mathrm{R}_{\mathrm{f}} 0.86\left(\mathrm{CHCl}_{3}\right.$ : Ethylacetate $=9: 1) ; \mathrm{IR}(\mathrm{KBr}) v_{\max } / \mathrm{cm}^{-1}: 1719 ;{ }^{1} \mathrm{HNMR}$ $\left(400 \mathrm{MHz}\right.$, DMSO- $\left.d_{6}\right) \delta 3.0(\mathrm{~s}, 3 \mathrm{H}), 7.0-7.3(\mathrm{~m}, 5 \mathrm{H}), 7.4$ (t, J $6.4 \mathrm{~Hz}, 2 \mathrm{H},), 7.6(\mathrm{~d}, J 8.3 \mathrm{~Hz}, 1 \mathrm{H}),, 8.2(\mathrm{~d}, J 8.3 \mathrm{~Hz}$, $1 \mathrm{H},), 8.5(\mathrm{~d}, J 1.5 \mathrm{~Hz}, 1 \mathrm{H}),, 8.6(\mathrm{dd}, J 7.7$ and $1.9 \mathrm{~Hz}, 1 \mathrm{H})$, 9.6 (bs, $1 \mathrm{H}, \mathrm{NH}) ;{ }^{13} \mathrm{CNMR}\left(75 \mathrm{MHz}, \mathrm{CDCl}_{3}\right) \delta 39.9\left(\mathrm{CH}_{3}\right)$, $115.7(\mathrm{CH}), 119.1(\mathrm{CH}), 120.9(\mathrm{CH}), 121.9(\mathrm{CH}), 125.0$ $(\mathrm{CH}), 127.7(\mathrm{C}), 128.2(\mathrm{C}), 129.7(\mathrm{CH}), 130.3(\mathrm{CH}), 132.9$ (C), 135.8 (C), 147.3 (C), 152.0 (C), 155.0 (C), 164.5 (C); 
MS $453\left(\mathrm{M}^{+}, 100 \%\right)$; Elemental analysis found: C, 55.57, $\mathrm{H}$ 3.30, N 9.31; $\mathrm{C}_{21} \mathrm{H}_{15} \mathrm{~N}_{3} \mathrm{O}_{7} \mathrm{~S}$ requires $\mathrm{C}, 55.63 ; \mathrm{H}, 3.33$; $\mathrm{N}, 9.27 \%$.

$N$-[4-(2,5-dioxo-2,5-dihydropyrrol-1-yl)-2-phenoxyphenyl] methanesulfonamide (3d)

Pale yellow solid; $\mathrm{mp} 145-148{ }^{\circ} \mathrm{C} ; \mathrm{R}_{\mathrm{f}} 0.80\left(\mathrm{CHCl}_{3}\right.$ : Ethylacetate $=9: 1) ; \mathrm{IR}(\mathrm{KBr}) v_{\max } / \mathrm{cm}^{-1}: 1725,1708 ;{ }^{1} \mathrm{HNMR}$ $\left(400 \mathrm{MHz}, \mathrm{CDCl}_{3}\right) \delta 3.0(\mathrm{~s}, 3 \mathrm{H}), 6.80$ (s, 2H), 6.88 (d, J 6.0 $\mathrm{Hz}, 1 \mathrm{H}), 6.96$ (bs, 1H, NH), 7.05 (d, J $9.0 \mathrm{~Hz}, 2 \mathrm{H}), 7.12$ $(\mathrm{dd}, J 12.0 \mathrm{~Hz}, J 6.0 \mathrm{~Hz}, 1 \mathrm{H}), 7.18(\mathrm{t}, J 9.0 \mathrm{~Hz} 1 \mathrm{H}), 7.40$ (t, $J 12.0 \mathrm{~Hz}, 2 \mathrm{H}), 7.75(\mathrm{~d}, J 12.0 \mathrm{~Hz}, 1 \mathrm{H}) ;{ }^{13} \mathrm{CNMR}(75 \mathrm{MHz}$, $\left.\mathrm{CDCl}_{3}\right) \delta 39.7\left(\mathrm{CH}_{3}\right), 115.6(\mathrm{CH}), 118.9(\mathrm{CH}), 121.2(\mathrm{CH})$, $121.6(\mathrm{CH}), 124.7(\mathrm{CH}), 127.5(\mathrm{C}), 128.2(\mathrm{C}), 130.2(\mathrm{CH})$, $134.1(\mathrm{CH}), 147.2(\mathrm{C}), 155.2(\mathrm{C}), 169.0(\mathrm{CO})$; MS $359\left(\mathrm{M}^{+}\right.$, 100\%); Elemental analysis found: C, 56.90, H 3.96, N 7.97; $\mathrm{C}_{17} \mathrm{H}_{14} \mathrm{~N}_{2} \mathrm{O}_{5} \mathrm{~S}$ requires $\mathrm{C}, 56.98 ; \mathrm{H}, 3.94 ; \mathrm{N}, 7.82 \%$.

N-[4-(2,5-dioxo-pyrrolidin-1-yl)-2-phenoxyphenyl]methanesulfonamide (3e)

Off white solid; mp $225-228{ }^{\circ} \mathrm{C} ; \mathrm{R}_{\mathrm{f}} 0.33\left(\mathrm{CHCl}_{3}\right.$ : Ethylacetate $=9: 1) ; \mathrm{IR}(\mathrm{KBr}) v_{\max } / \mathrm{cm}^{-1}: 1707 ;{ }^{1} \mathrm{HNMR}(300$ $\left.\mathrm{MHz}, \mathrm{CDCl}_{3}\right) \delta 2.7$ (s, 4H), 3.1 (s, 3H), 6.8 (s, 1H), 7.1( $\mathrm{m}, 3 \mathrm{H},), 7.3$ (m, 1H), 7.4 (m, 3H), 9.5 (s, NH,); ${ }^{13} \mathrm{CNMR}$ $\left(75 \mathrm{MHz}, \mathrm{CDCl}_{3}\right) \delta 28.3\left(\mathrm{CH}_{2}\right), 39.8\left(\mathrm{CH}_{3}\right), 115.9(\mathrm{CH})$, $119.1(\mathrm{CH}), 120.8(\mathrm{CH}), 122.1(\mathrm{CH}), 124.8(\mathrm{CH}), 128.1(\mathrm{C})$, $128.6(\mathrm{C}), 130.2(\mathrm{CH}), 147.2(\mathrm{C}), 155.1(\mathrm{C}), 175.7(\mathrm{CO})$; MS $361\left(\mathrm{M}^{+}, 100 \%\right)$; Elemental analysis found: C, 56.70, $\mathrm{H} 4.50, \mathrm{~N} 7.74 ; \mathrm{C}_{17} \mathrm{H}_{16} \mathrm{~N}_{2} \mathrm{O}_{5} \mathrm{~S}$ requires $\mathrm{C}, 56.66 ; \mathrm{H}, 4.47$; $\mathrm{N}, 7.77 \%$.

$N$-[4-(2,6-dioxo-piperidin-1-yl)-2-phenoxyphenyl]methanesulfonamide (3f)

White solid; mp $126-128{ }^{\circ} \mathrm{C} ; \mathrm{R}_{\mathrm{f}} 0.38\left(\mathrm{CHCl}_{3}\right.$ : Ethylacetate $=1: 1)$; IR $(\mathrm{KBr}) \mathrm{v}_{\max } / \mathrm{cm}^{-1}: 1671 ;{ }^{1} \mathrm{HNMR}$ $\left(400 \mathrm{MHz}, \mathrm{DMSO}-d_{6}\right) \delta 1.7(\mathrm{~m}, 2 \mathrm{H}), 2.4(\mathrm{~m}, 4 \mathrm{H}), 3.0(\mathrm{~s}$, $3 \mathrm{H}), 7.0(\mathrm{dd}, J 8.8$ and $1 \mathrm{~Hz}, 2 \mathrm{H}), 7.2-7.3(\mathrm{~m}, 4 \mathrm{H}), 7.42$ $(\mathrm{m}, 2 \mathrm{H}), 10.0(1 \mathrm{H}, \mathrm{NH}) ;{ }^{13} \mathrm{CNMR}\left(75 \mathrm{MHz}, \mathrm{DMSO}-d_{6}\right)$ $\delta 20.2\left(\mathrm{CH}_{2}\right), 32.9\left(\mathrm{CH}_{2}\right), 35.3\left(\mathrm{CH}_{2}\right), 108.6(\mathrm{CH}), 113.8$ $(\mathrm{CH}), 119.1(\mathrm{CH}), 122.3(\mathrm{C}), 123.8(\mathrm{CH}), 127.8(\mathrm{CH})$, $129.9(\mathrm{CH}), 138.3(\mathrm{C}), 151.0(\mathrm{C}), 155.9(\mathrm{C}), 170.7(\mathrm{C})$, $174.0(\mathrm{CO})$; Mass $375\left(\mathrm{M}^{+}, 100 \%\right)$; Elemental analysis found: $\mathrm{C}, 57.69, \mathrm{H} 4.86, \mathrm{~N} 7.52 ; \mathrm{C}_{18} \mathrm{H}_{18} \mathrm{~N}_{2} \mathrm{O}_{5} \mathrm{~S}$ requires $\mathrm{C}$, $57.74 ; \mathrm{H}, 4.85 ; \mathrm{N}, 7.48 \%$.

\section{Anti-inflammatory activity}

The anti-inflammatory activity of five compounds (3a, $\mathbf{3 b}, \mathbf{3 c}, \mathbf{3 e}$ and $\mathbf{3 f}$ ) was evaluated according to the method reported in the literature ${ }^{23}$ where a pedal inflammation in rat paws was induced by sub plantar injection of $0.1 \mathrm{~mL}$ carrageenan $(0.2 \%)$ suspension in gum acacia into the right hind of the rats. Male adult albino Wister rats (100$120 \mathrm{~g}$ ) were divided into 12 groups of six animals each. The rat paw thickness was measured with a Veriner caliper before and $1 \mathrm{~h}$ after the carrageenan injection to detect the carrageenan induced inflammation. Each test compound at a dose of $100 \mathrm{mg} \mathrm{kg}^{-1}$ was injected i.p. to a separate group of rats $1 \mathrm{~h}$ after carrageenan injection. Control group received the vehicle (5\% gum acacia), while the reference group received indomethacin, $10 \mathrm{mg} \mathrm{kg}^{-1}$.

The difference between the thicknesses of the two paws was taken as a measure of edema. The measurement was carried out at zero, 1, 2, 3 and $4 \mathrm{~h}$ after injection of the test compounds, reference drug, and the vehicle.

\section{Acknowledgments}

The authors (K. Kavitha and S. Pal) thank Mr. M. N. Raju, the chairman of MNR Educational Trust for his support and encouragement.

\section{References}

1. Salvati, M. E.; Balog, A.; Shan, W.; Wei, D. D.; Pickering, D.; Attar, R. M.; Geng, J.; Rizzo, C. A.; Gottardis, M. M.; Weinmann, R.; Krystek, S. R.; Sack, J.; An, Y.; Kish, K.; Bioorg. Med. Chem. Lett. 2005, 15, 271.

2. Kossakowski, J.; Jarocka, M.; Il Farmaco 2001, 56, 785; Sondhi, S. M.; Rani, R.; Roy, P.; Agarwal, S. K.; Saxena, A. K.; Bioorg. Med. Chem. Lett. 2009, 19, 1534.

3. Ishizumi, K.; Kojma, A.; Antoku, F.; Chem. Pharm. Bull. 1991, 39, 2288.

4. Shibata, Y.; Shiehita, M.; Sasaki, K.; Nishimura, I. L.; Hashimoto, Y.; Iwasaki, S.; Chem. Pharm. Bull. 1995, 43, 177.

5. Jindal, D. P.; Bedi, V.; Jit, B.; Karkra, N.; Guleria, S.; Bansal, R.; Palusczak, A.; Hartmann, R.W.; Il Farmaco 2005, 60, 283.

6. Wang, J. J.; Wang, S. S.; Lee, C. F.; Chung, M. A.; Chern, Y. T.; Chemotherapy 1997, 43, 182.

7. Machado, A. L.; Lima, L. M.; Arau’jo, Jr., J. X.; Fraga, C. A. M.; Koatz, V. L. G.; Barreiro, E. J.; Bioorg. Med. Chem. Lett. $\mathbf{2 0 0 5}, 15,1169$.

8. Kenji, S.; Hideko, N.; Yoshihiro, U.; Yoshikazu, S.; Kazuharu, N.; Motoji, W.; Konstanty, W.; Tadafumi, T.; Tetsuji, A.; Yuji, Y.; Kenji, K.; Hitoshi, H.; Bioorg. Med. Chem. 2005, 13, 4014.

9. Mayer, A.; Neuenhofer, S.; Angew. Chem., Int. Ed. 1994, 33, 1044.

10. Miguel, F. B.; Gema, D.; Beatriz, S.; Cynthia, R.; Simmon, R.; Teresa, B.; Eur. J. Med. Chem. 2002, 37, 541.

11. Miyachi, H.; Azuma, A.; Ogasawara, A; Uchimura, E.; Watanabe, N.; Kobayashi, Y.; Kato, F.; Kato, M.; Hashimoto, H.; J. Med. Chem. 1997, 40, 2858. 
12. Pericherla, S.; Mareddy, J.; Rani, D. P. G.; Gollapudi, P. V.; Pal, S.; J. Braz. Chem. Soc. 2007, 18, 384.

13. Walker, M. A.; J. Org. Chem. 1995, 60, 5352; Walker, M. A.; Tetrahedron Lett. 1994, 35, 665.

14. Dorta, R. L.; Francisco, C. G.; Sua'rez, E.; Tetrahedron Lett. 1994, 35, 1083.

15. Da Settimo, A.; Primofiore, G.; Settimo, F. Da; Simorini, F.; LaMotta, C.; Martinelli, A.; Boldrini, E.; Eur. J. Med. Chem. 1996, 31, 49.

16. Mehta, N. B.; Phillips, A. P.; Lui, F. F.; Brooks, R. E.; J. Org. Chem. 1960, 25, 1012.

17. Reddy, P. Y.; Kondo, S.; Toru, T.; Ueno, Y.; J. Org. Chem. 1997, 62, 2652.

18. Abdel-Aziz, A. A.-M.; Eur. J. Med. Chem. 2007, 42, 614.

19. Bose, A. K.; Manhas, M. S.; Ghush, M.; Raju, V. S.; Tabei, K.; Urbanczyk-Lykewaska, Z.; Heterocycles 1990, 30, 741.
20. Westaway, K. C.; Gedye, R. N.; J. Microw. Power Electromagn. Energy 1995, 30, 219.

21. Chandrasekhar, S.; Takhi, M.; Uma, G.; Tetrahedron Lett. 1997, $38,8089$.

22. Akula, M. R.; Kabalka, G. W.; Synth. Commun. 1998, $28,2063$.

23. Nargund, L. V. G.; Reedy, G. R. N.; Haripbasad, V.; J. Pharm. Sci. 1994, 83, 246.

24. For the synthesis of cyclic unsubstituted imides, see: Mederski, W. W. K. R.; Baumgarth, M.; Germann, M.; Kux, D.; Weitzel, T.; Tetrahedron Lett. 2003, 44, 2133; Hijji, Y.; Benjamin, E.; Heterocycles 2006, 68, 2259.

Received: December 22, 2009 Web Release Date: March 8, 2010 